\title{
EL CAMINO HACIA LA PATENTACIÓN EN LAS UNIVERSIDADES
}

\section{THE WAY TOWARDS THE PATENTS IN THE UNIVERSITIES}

\author{
Rodolfo Schmal S. ${ }^{1} \quad$ María del Socorro López G. $^{2} \quad$ Fernando Cabrales G. $^{3}$
}

Recibido 9 de enero de 2006, aceptado 30 de agosto de 2006

Received: January 9, 2006 Accepted: August 30, 2006

\begin{abstract}
RESUMEN
En los últimos 25 años se le ha dado importancia creciente a la producción de tecnología y otras formas de creación de conocimientos con valor comercial en las universidades en todo el mundo. En este trabajo se evalúa el desempeño de las universidades chilenas como productoras de conocimientos con valor comercial restringido a la propiedad intelectual con aplicaciones industriales en los últimos 10 años. La expresión cuantificable de esto son las patentes industriales, los modelos de utilidad y los diseños industriales. Además se realiza una síntesis de la forma en que están incorporando este aspecto del trabajo académico las principales universidades chilenas. Finalmente se comparan los resultados relativos observados en Chile con los obtenidos en EE.UU. y Europa.
\end{abstract}

Palabras clave: Patentes, universidad, derechos de propiedad intelectual, innovación, investigación, tecnología.

\begin{abstract}
In the last 25 years, growing importance has been given to technology production and other forms of knowledge creation, that is produced by universities throughout the world and has commercial value. In this paper, the last ten years' performance of Chilean universities as producers of knowledge with commercial value, and industrial applications, as well as restricted to intellectual property, are evaluated. Industrial patents, models of utility and industrial designs are considered as quantifiable referents. A synthesis of the way the main Chilean universities are incorporating this aspect of the academic work is presented. Finally, the relative results observed in Chile are compared with those obtained in the USA and Europe.
\end{abstract}

Keywords: Patents, university, intellectual property rights, innovation, research, technology.

\section{INTRODUCCIÓN}

En un escenario mundial donde el conocimiento ha pasado a ser uno de los principales activos de las empresas, el concepto de propiedad intelectual ha surgido como un factor clave para proveer el marco regulador y los incentivos apropiados que atraigan la inversión para innovar. Se trata de recompensar el esfuerzo que demanda toda innovación, tras la cual se requieren crecientes recursos financieros, por la vía de la obtención de una patente que represente una propiedad tangible, susceptible de transarse comercialmente.

Las universidades, como instituciones responsables de la creación, transmisión y difusión del conocimiento, han jugado y deben seguir jugando un papel fundamental en la provisión de conocimiento como bien público $[6,15,18]$.

Históricamente la relación entre la ciencia y la tecnología ha transitado desde una virtual independencia mutua hasta la realidad actual, marcada por una creciente interdependencia y una progresiva simbiosis entre ambas donde la actividad tecnológica se hace cada vez más dependiente del conocimiento científico.

Mientras la tecnología está centrada en el "saber cómo" (know how), la ciencia lo está en el "saber por qué" (know why), de allí que la "ciencia” sólo es más abstracta, metodológicamente más rigurosa y se sitúa en la frontera del conocimiento, muchas veces por los problemas e hipótesis que surgen de la propia tecnología que ha producido o como

\footnotetext{
1 Facultad de Ciencias Empresariales, Universidad de Talca. 2 Norte 685, Casilla 721, Talca-Chile; rschmal@utalca.cl

2 Facultad de Ciencias Económicas, Universidad de Antioquia. Calle 67 \#53-108 Bl 13, Medellín-Colombia; mslopez@agustinianos.udea.edu.co

3 Facultad de Ciencias Sociales, Administrativas y Económicas, Universidad de Tarapacá. 18 de Septiembre 222, Arica-Chile; fcabrale@uta.cl
} 
insumo de nuevos problemas que se plantean en las diversas disciplinas. La separación analítica entre una y otra es útil sólo para los propósitos de "gestión de conocimientos" [13] pues la tecnología está relativamente cerca de productos o servicios comerciales y en ese sentido debe administrarse de manera diferenciada a la "ciencia".

Sin embargo, la interrelación cada vez mayor entre la ciencia y la tecnología ha generado el concepto de conocimiento científico tecnológico y el surgimiento de nuevas ciencias tales como la robótica, la biotecnología, la nanotecnología, entre otras, que están exigiendo a las universidades incorporar en su misión la solución de problemas específicos, los cuales requieren un alto componente de investigación aplicada.

Además la intensificación del comercio (especialmente el internacional) y el aumento de la participación de los servicios, por definición intangibles y muchas veces incorporados en los bienes, en el producto agregado mundial están aumentando las exigencias sobre la transparencia de los mercados (normalización, certificación, etc.) y, notablemente, la "titularización" de las ideas e innovaciones. De hecho, la OCDE calcula que entre 1985 y 1997 la contribución de industrias basadas en el conocimiento al valor agregado total aumentó desde 51 hasta $59 \%$ en Alemania y desde 45 hasta $51 \%$ en Inglaterra. Además sólo en EE.UU. los ingresos por licencias se han multiplicado por 10 desde 1990 al 2000, desde US\$ 10.000 millones a US\$ 100.000 millones y en toda la OECD la inversión corporativa privada en I+D ha aumentado en un $51 \%$ en el mismo período, ganando más de 0,5 puntos porcentuales en su participación en el PIB [17], entre muchas otras cifras que muestran la creciente importancia de la tecnología como motor del crecimiento.

Estos aspectos así como otros relacionados con el desarrollo regional [11] han inducido tanto a las universidades como a la empresa privada y al Estado a estrechar sus vínculos para el desarrollo tecnológico e industrial y a convertir a las universidades en protagonistas principales del cambio tecnológico.

De allí que en la actualidad el énfasis esté puesto en la necesidad de vincular más estrechamente la investigación universitaria con las necesidades de empresas, las que se encuentran bajo el desafío de incorporar mayor valor a sus productos/servicios. En un contexto de creciente relevancia de los derechos de propiedad intelectual y de incorporar nuevas fuentes de financiamiento para la investigación, Estados Unidos con la Ley Bayh-Dole (1980) ha dado vía libre a las universidades para que comercialicen los resultados de la investigación financiada con dineros públicos, estableciendo un hito mundial en el concepto y propósito de la $\mathrm{I}+\mathrm{D}$ universitaria, cuyos resultados se habían considerado hasta entonces fundamentalmente como bienes públicos, no susceptibles de explotarse comercialmente, y sólo valorados académicamente.

Mientras se produce abundante literatura que analiza desde diversas perspectivas el impacto de dicha ley $[10,19,21]$, las universidades estadounidenses se han convertido en los últimos 25 años en grandes productores de patentes y los países europeos adecuan sus legislaciones en esta misma vía con resultados parecidos. De hecho, el cambio del dinamismo tecnológico de la UE y EE.UU. está asociado a la producción tecnológica de sus universidades. Ello ha traído también abundantes encadenamientos productivos en la "economía del conocimiento" pues el crecimiento de la producción comercial de las universidades está también asociado a la proliferación de parques tecnológicos, incubadoras de empresas y asociaciones de diverso tipo con las empresas [4, 12, 20, 22]. Las universidades latinoamericanas, aunque no tienen tradición de patentar, no pueden eximirse de evaluar en profundidad este cambio de contexto, particularmente cuando los tratados de libre comercio (TLC) que están suscribiendo los países latinoamericanos con Estados Unidos les obliga a ajustar sus marcos jurídicos a los convenios internacionales en materia de Derechos de Propiedad Intelectual y específicamente en el campo de las patentes. Incluso por encima de dichos convenios (los llamados plus en el acuerdo sobre los Aspectos de la Propiedad Intelectual Relacionados con el Comercio -ADPIC-) obliga a los países a renunciar a las salvaguardas allí establecidas $\mathrm{y} / \mathrm{o}$ a productos y procedimientos que no son objetos de patentación, para que sean patentables.

De hecho, la universalización de la legislación sobre propiedad intelectual puede verse, además de una imposición de los tiempos, como una oportunidad para los países en desarrollo para participar de un negocio de grandes perspectivas. En este contexto las universidades chilenas tienen que dimensionar su responsabilidad en el desarrollo técnico del país con el apoyo al Estado en la construcción de políticas y estrategias para la aplicación de los derechos de propiedad intelectual, al tiempo que se preparan para incursionar por la senda de patentar los resultados de sus programas de investigación.

Para abordar el presente trabajo se realizó un estudio exploratorio que involucró:

a) entrevistas y reuniones con quienes tienen la responsabilidad de la patentación en Chile 
(Departamento de Propiedad Industrial del Ministerio de Economía) y de algunas de sus universidades (Vicerrectorías y Direcciones de Investigación);

b) revisión bibliográfica acerca de la legislación en materia de derechos de propiedad intelectual;

c) recopilación de estadísticas nacionales e internacionales relativas a la producción de patentes;

d) recopilación de antecedentes relativos a las políticas que se están aplicando para proteger la creación de conocimientos en las universidades.

\section{MARCO INSTITUCIONAL}

En Chile las normas aplicables a los privilegios industriales y protección de los derechos de propiedad industrial están contenidas en la ley 19.039 del año 1991 sobre Propiedad Industrial. Recientemente, en marzo del presente año, se publicó la ley 19.996 que introduce cambios con miras a adecuar la legislación vigente a los estándares que la Organización Mundial del Comercio (OMC) estableció en el acuerdo sobre los ADPIC. Esta nueva ley, vigente desde diciembre de 2005 y que cambia varios aspectos importantes de la legislación chilena sobre la propiedad industrial, está siendo examinada por la OMC para verificar su conformidad con el acuerdo ADPIC. En ella se incluyen cambios que conciernen fundamentalmente a nuevas categorías de derechos como denominaciones de origen, circuitos integrados, secreto empresarial y procedimientos civiles a seguir en caso de vulnerarse los derechos de propiedad intelectual.

Esta ley es implementada en un contexto mundial en el que se ha producido un desarrollo tecnológico sin precedentes; y un contexto nacional, que en lo fundamental y en relación a los países de mayor desarrollo, de acuerdo a información de la Comisión Nacional de Investigación Científica y Tecnológica (CONICYT) y la Red de Indicadores de Ciencia y Tecnología Iberoamericana e Interamericana (RICYT), se ha caracterizado por:

- Un bajo volumen de gasto en investigación y desarrollo (I+D), el cual no supera el 0.7\% del PIB, en circunstancias que en los países desarrollados el gasto en este ítem está sobre el $2 \%$ de un PIB significativamente mayor;

- Una escasa participación privada en el financiamiento del gasto en $\mathrm{I}+\mathrm{D}$, por debajo del $50 \%$ del gasto, en tanto que en los países desarrollados su incidencia está muy por encima del gasto público en $\mathrm{I}+\mathrm{D}$;
- Una concentración del gasto en I+D en las universidades, lo que conlleva un bajo gasto por este concepto en las empresas productivas; $y$

- Un esfuerzo investigativo centrado en las ciencias básicas o puras, antes que en ciencias aplicadas o desarrollo tecnológico o creación de nuevos productos y procedimientos como ocurre en los países de mayor desarrollo.

Para superar esta realidad, con miras a mejorar la competitividad del país, se ha planteado la necesidad de elaborar estrategias basadas en fortalecer las actividades de I+D; promover la transferencia tecnológica; y potenciar la vinculación de las universidades con el sector productivo.

Para encarar estos desafíos, adquieren relevancia los temas asociados a la propiedad intelectual. Los factores que están incidiendo en ello son:

a) La firma de múltiples tratados de libre comercio, los que incluyen capítulos sobre la materia por las cuales Chile se obliga a suscribir una serie de tratados internacionales en materia de propiedad intelectual;

b) la existencia de un nuevo marco legal expresado en leyes vinculadas a mercaderías importadas que no respetan los derechos de propiedad intelectual (ley 19.912); a los derechos de autor para adecuarlos al tratado suscrito con EE.UU. (ley 19.914); y la modificación de la ley 19.039 para adecuarla al acuerdo ADPIC de la OMC (ley 19.996).

En Chile, el otorgamiento de los derechos de autor está bajo la competencia del Ministerio de Educación, en tanto que el otorgamiento de los títulos y demás servicios relativos a la propiedad industrial competen al Departamento de Propiedad Industrial, unidad dependiente del Ministerio de Economía y Energía a través de la Subsecretaría de Economía.

Por tanto, el Departamento de Propiedad Industrial es el único organismo que garantiza la protección de un derecho de propiedad sobre un objeto (una invención, un modelo de utilidad, un diseño industrial) o una marca, mediante la concesión de patentes y marcas. En la actualidad se encuentra en tramitación un proyecto de ley destinado a transformarlo en un Instituto de Propiedad Industrial para dotar al Estado de una institucionalidad capaz de resolver aspectos relacionados con la tramitación y concesión de derechos; de informar acerca de las alternativas tecnológicas existentes; y de la promoción de la protección de la propiedad industrial. 


\section{LAS PATENTES}

Jurídicamente una patente es un derecho de exclusividad concedido por el Estado para proteger y explotar un objeto, por un período de tiempo. Una patente es la contrapartida al hecho de proteger un objeto mediante reserva o secreto $\mathrm{y}$, como tal, requiere que el solicitante haga público su invento a través de una solicitud de patente, de forma que un experto en la materia pueda reproducir el objeto. A cambio de hacer público su objeto, el solicitante obtiene un derecho que permite impedir a terceros cualquier forma de explotación comercial del objeto, dentro del territorio de obtención de la patente y por un periodo de tiempo limitado.

Si bien la legislación sobre patentes en Chile es semejante a la mayoría de los demás países occidentales, tiene algunas particularidades importantes. En Chile una patente de invención se concedía por un período no renovable de 15 años, período recientemente aumentado a 20 años, en lo que parece una señal del interés por reforzar los incentivos para patentar ideas con valor industrial [16]. Cabe señalar que en la mayor parte del resto del mundo este período es de 20 años, el plazo máximo acordado por el ADPIC, contados desde la fecha de otorgamiento. En cambio una patente de modelo de utilidad o de diseño industrial es concedida por 10 años contabilizados desde la fecha de presentación de la solicitud correspondiente.

Para la obtención de una patente, el objeto (invención, modelo de utilidad y diseño industrial) a proteger debe cumplir las siguientes características adicionales a las consideradas en la definición de ellos:

- Ser novedoso, lo que implica que a la fecha de presentación de la solicitud de patente no se encuentra accesible bajo ninguna forma;

- Tener nivel inventivo, lo que implica que no resulta obvio ni evidente bajo el actual estado del arte en la materia para quienes se desempeñan en el área correspondiente; y

- Ser factible de reproducir o utilizar en el sector productivo que corresponda (manufacturero, minero, agrícola u otros).

El tiempo de tramitación de una patente, desde que es solicitada hasta que es concedida, es del orden de 6 años en el caso de las patentes de invención, de 5 años para las patentes de modelos de utilidad y de 3 años para las patentes de diseño industrial.
La concesión de una patente permite a su titular:

- Ser reconocido por su creatividad y obtener recompensas materiales por sus objetos comercializables;

- Decidir quién(es) puede(n) utilizar el objeto patentado durante el periodo en el que se encuentra protegido;

- Vender la titularidad de la patente a un tercero, esto es, el derecho sobre el objeto involucrado;

- Impedir la copia o imitación de los objetos patentados por parte de terceros (competidores); y

- Otorgar una licencia (o permiso) a terceros para utilizar el objeto en los términos establecidos de común acuerdo.

Todo titular de una patente, a cambio de la protección, debe publicar la información sobre su objeto, con el fin de enriquecer el conocimiento técnico existente, debiendo evitar divulgar previamente el objeto a patentar (pues rompería uno de los requisitos exigidos: la novedad). Esta imposibilidad de difusión, en el caso de los investigadores universitarios se contrapone con lo que ha sido habitual en Chile, puesto que en general los investigadores son evaluados por las publicaciones antes que por las patentes que sus proyectos de investigación logren generar. Este aspecto (junto a otros característicos de la actividad académica) ha sido largamente estudiado en el caso de economías más desarrolladas pues obliga a optar muy tempranamente a los investigadores entre proyectos "científicos" (cuyo objetivo es la publicación) y los técnico-comerciales (cuyo objetivo es la patente).

Las ventajas que suelen endosarse a un sistema de patentación se centran en:

- Permiten determinar el estado de avance de una determinada tecnología evitando la duplicidad de esfuerzos en materia de $\mathrm{I}+\mathrm{D}$, constituyéndose en un medio de divulgación tecnológico de más reciente publicación que abarca a todos los sectores respecto de lo nuevo aplicable a la industria y relevante a nivel internacional.

- Facilitan la búsqueda de socios comerciales y de soluciones existentes para resolver problemas tecnológicos específicos vía soluciones susceptibles de ser implementadas a través de negociaciones de licencias al ordenar bajo un sistema de Clasificación Internacional de Patentes (CIP) único que permite identificar las patentes por área específica de la técnica. 
- Determinan las posibilidades de patentar una tecnología en particular evitando vulnerar una ya protegida por una patente, infracción que puede ser de alto costo.

- Posibilitan la recuperación de las inversiones que demandan las innovaciones apoyando la transferencia tecnológica tanto desde el ámbito de la investigación al sector productivo como desde los países desarrollados hacia los de menor desarrollo.

- Describen el objeto de manera clara y completa e identifican a su inventor facilitando la localización de posibles fuentes tecnológicas, el conocimiento del entorno competitivo de un mercado, el seguimiento de las actividades de una determinada empresa o el acceso a una determinada licencia.

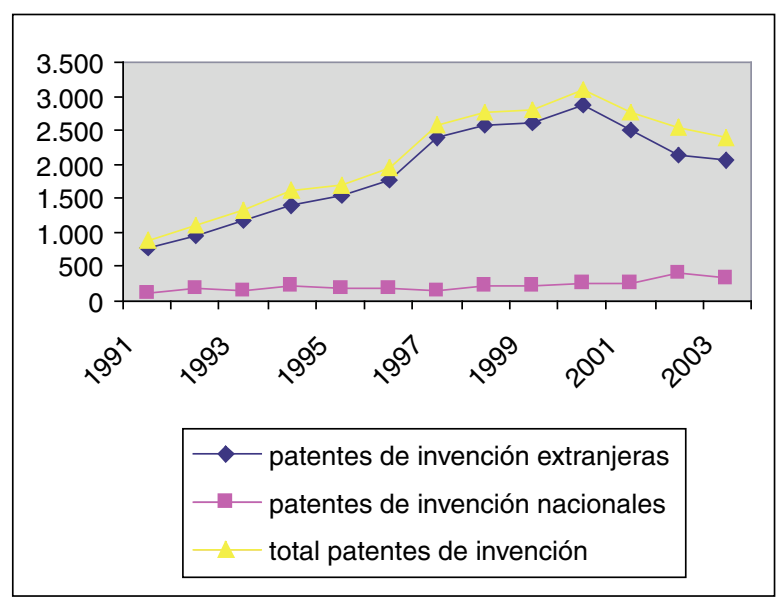

Gráfico 1a) Solicitudes de patentes.

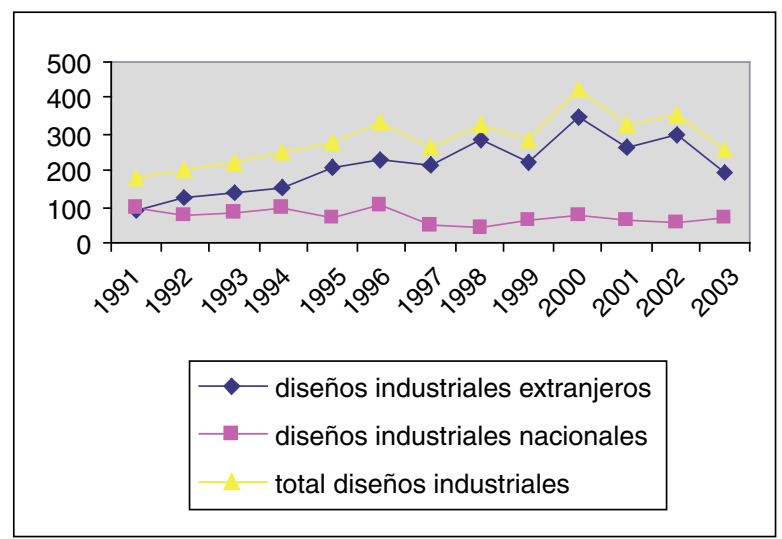

Gráfico 1c) Solicitudes de diseños industriales.

\section{ANÁLISIS DE LA PRODUCCIÓN DE PATENTES UNIVERSITARIAS EN CHILE}

\section{La producción agregada de Derechos de Propiedad Industrial en Chile}

Los datos sobre la producción de patentes, modelos de utilidad y diseños industriales en Chile se obtuvieron desde el sitio web de la Oficina de Información del Departamento de Propiedad Industrial del Ministerio de Economía (OFINTEC, www.dpi.cl). La evolución de las patentes, modelos de utilidad y diseños industriales solicitadas en Chile según el lugar de residencia de los solicitantes se muestran en los gráficos 1a, 1b, 1c y 1d.

Se puede observar que las patentes solicitadas por chilenos en Chile son una proporción pequeña y decreciente del

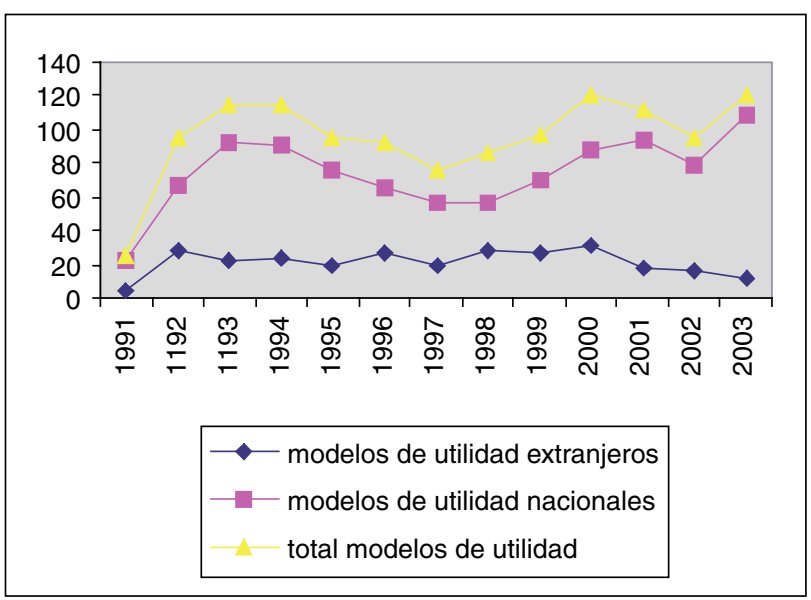

Gráfico 1b) Solicitudes de modelos de utilidad.

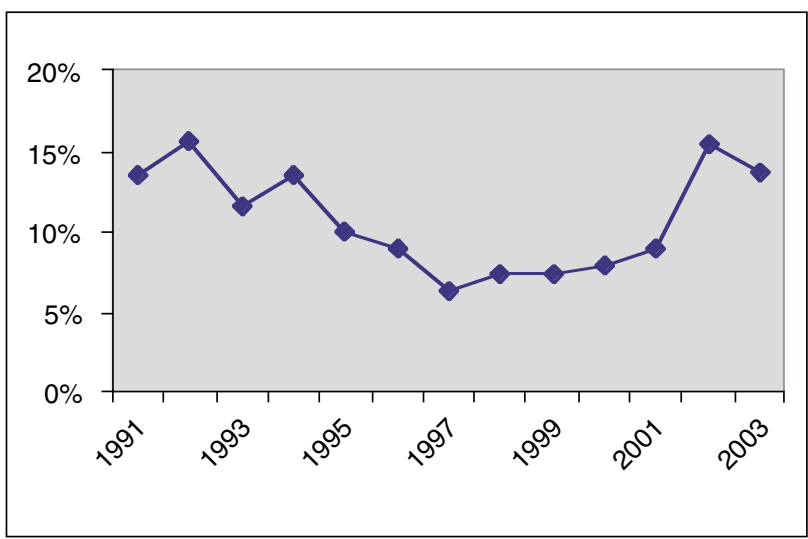

Gráfico 1d) Porcentajes de solicitudes de patentes de invención nacionales sobre el total de solicitudes.

Fuente: Elaboración propia a partir de datos de OFINTEC. 
total de solicitudes realizadas en nuestro país (gráfico 1d). En el gráfico la se observa que el comportamiento dinámico de las solicitudes está determinado por las solicitudes de no residentes. Las de nacionales varían poco en los 10 años analizados.

En el caso de los modelos de utilidad (o patentes menores en la terminología de Propiedad Industrial) el comportamiento de las solicitudes nacionales es más relevante respecto del total, pero la producción de este tipo de propiedad industrial es cuantitativa y cualitativamente (por definición) menos significativa. Se puede suponer que este comportamiento se debe precisamente a la menor importancia de los modelos de utilidad pues para los extranjeros resulta menos rentable solicitar uno de estos derechos de propiedad industrial (en adelante DPI) considerando que tienen plazos de protección más cortos y calidad inventiva menos radical.

En el caso de los diseños industriales hay una situación intermedia pues las solicitudes nacionales vuelven a ser

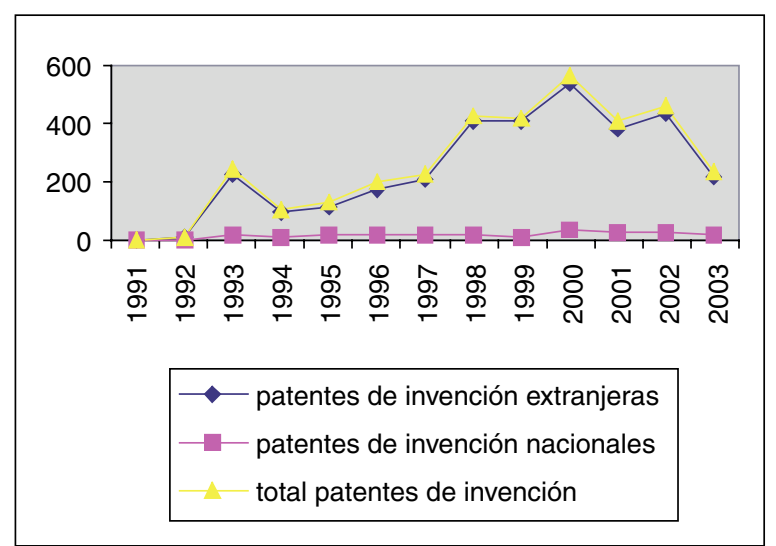

Gráfico 2a) Patentes concedidas.

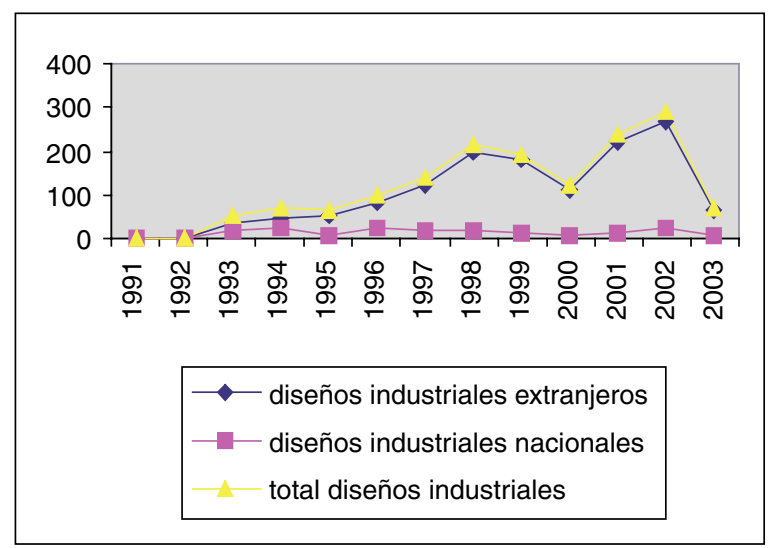

Gráfico 2c) Diseños industriales concedidos.

Fuente: Elaboración propia a partir de datos de OFINTEC. menos importantes proporcionalmente y el número de solicitudes es menor que el de las patentes pero mayor que las de los modelos de utilidad.

En cuanto a las patentes, modelos de utilidad y diseños industriales obtenidos, en el período analizado los datos se muestran en los gráficos $2 \mathrm{a}, 2 \mathrm{~b}, 2 \mathrm{c}$ y $2 \mathrm{~d}$.

Obsérvese que la concesión de DPIs en Chile sigue un patrón parecido a la solicitud de patentes, pero las cantidades involucradas resultan notoriamente inferiores aun considerando un período tan largo como 10 años. Resulta notable el dinamismo observado por las solicitudes a principios de los 90 y el consecuente aumento de las concesiones a mediados de los 90. Notamos que la Ley 19.039 es, precisamente, de 1991 de manera que la producción de DPIs parece tener un potencial importante en Chile que no parece desarrollarse (más bien parece frustrarse) por la escasa protección que proporcionaba la ley recientemente sustituida por la $\mathrm{N}^{\mathrm{o}} 19.996$.

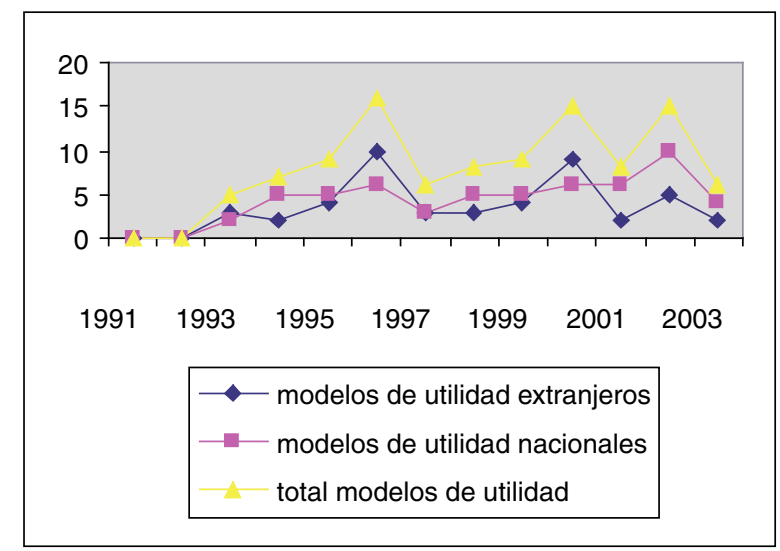

Gráfico 2b) Modelos de utilidad concedidos.

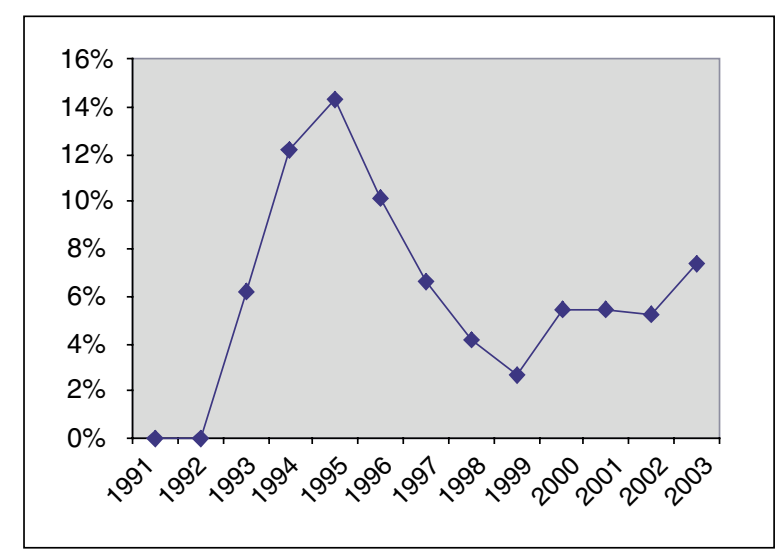

Gráfico 2d) Porcentaje de patentes concedidas a nacionales. 


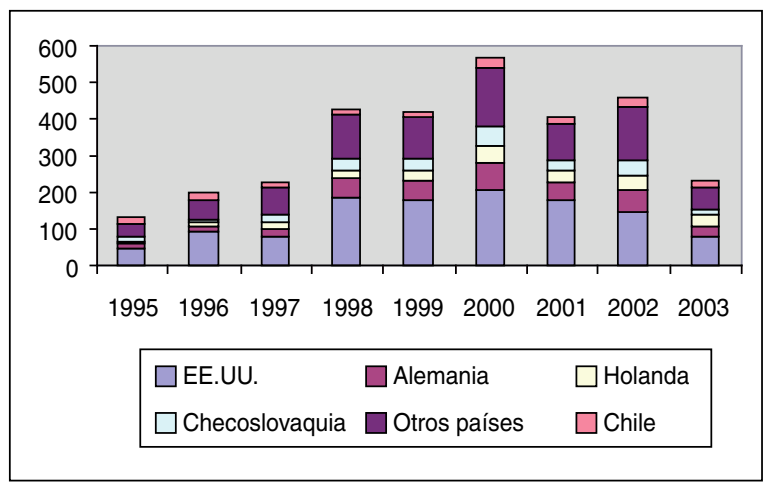

Fuente: Elaboración propia a partir de datos de OFINTEC.

Gráfico 3. Patentes concedidas en Chile por residencia del solicitante.

Un estudio interesante proviene de la comparación de nacionalidades de los titulares de patentes en Chile. El gráfico 3 muestra el país de residencia de los solicitantes de patentes obtenidas en Chile durante el período de estudio.

Se puede observar que los principales titulares de patentes en Chile son residentes de países de mayor desarrollo que el nuestro y pertenecientes principalmente a EE.UU. y la UE. Aun países como la República Checa y Eslovaquia (que se muestran juntos bajo la antigua denominación de Checoslovaquia) tienen mayor presencia que los propios chilenos. Esta comparación es relevante porque si se acepta que parte del bajo dinamismo productivo de patentes se debe a razones clásicas como: (a) El pequeño tamaño del mercado tecnológico chileno $[3,5]$. (b) La insuficiente protección del marco legal chileno [2]. (c) La ineficiencia del soporte administrativo de la protección industrial chilena [7], entonces es razonable suponer que aun cuando es escasa la producción de patentes, los agentes chilenos debieran tener una participación importante en el reducido mercado. Los datos muestran que ello no es así pues los residentes chilenos sólo ocupan un modesto quinto lugar a lo largo de 10 años.

\section{Las patentes universitarias en Chile}

En esta sección se presenta la producción de patentes universitarias a partir de datos proporcionados directamente por OFINTEC a los autores. Se desprende que la cantidad de solicitudes es proporcionalmente muy pequeña en relación al total nacional e incluso si se las compara sólo con las de residentes. Se observa que las universidades chilenas sólo han presentado 149 solicitudes de patentes en 10 años. Adicionalmente las universidades no exhiben un comportamiento regular en esta actividad. Sólo las universidades de Concepción y Técnica Federico Santa María solicitan regularmente patentes de invención.
Además entre ambas explican más del $50 \%$ de las solicitudes universitarias en Chile.

En el gráfico 4 se muestran las solicitudes de patentes realizadas por universidades chilenas en el período de análisis.

En la tabla 1 se presenta el estado en que se encuentran las patentes solicitadas por las universidades en el período considerado (1995 a 2004).

Del total de 149 patentes solicitadas en el período en estudio, solo se concedieron 7 patentes ( 2 a la U. de Concepción en 1998 y 1999; una a la U. de Santiago en 1998; una a la U. de Chile en 1996; una a la U. Austral en 1998; una a la U. Mayor en 1999, y una a la U. Católica del Norte en 1998.

Analizando el comportamiento de las patentes universitarias solicitadas y concedidas se observa que:

- Casi el $50 \%$ de las solicitudes están centradas en dos universidades: la Universidad de Concepción y la Universidad Técnica Federico Santa María, ambas creadas y con sus sedes centrales en regiones, Concepción y Valparaíso respectivamente;

- Casi el 90\% de las solicitudes son efectuadas por 7 universidades (U.T. F. Santa María, U. de Concepción, U. de Santiago, U. de Antofagasta, P. U. Católica, U. de Chile, U. Católica de Valparaíso), todas ellas pertenecientes al Consejo de Rectores;

- De todas las solicitudes, un 5\% de ellas fueron elevadas por una universidad en conjunto con otra institución, que ha sido otra universidad en todos los casos, con excepción de una de ellas, en el que la U. de Chile lo hizo con una empresa privada;

- Menos del 5\% de las patentes solicitadas fueron concedidas.

\section{Visión general de la gestión tecnológica universitaria chilena}

La producción de DPIs en las universidades parece de todos modos una inquietud latente en el sistema universitario chileno. Además la elaboración de un diagnóstico de sus éxitos y fracasos precisa reconocer la forma y características de las actividades conducentes a la producción de patentes y otros activos intelectuales.

Para caracterizar la gestión de la patentación en las universidades chilenas y conocer sus políticas en materia de patentamiento y derechos de propiedad intelectual, se efectuó un muestreo no probabilístico de un universo constituido por todas las universidades acreditadas 
Tabla 1. Estado de las patentes de invención solicitadas por las universidades.

\begin{tabular}{|c|c|c|c|c|c|}
\hline UNIVERSIDADES & En trámite & Abandonadas & Concedidas & Total & $\%$ \\
\hline U.T. F. Santa María & 18 & 4 & 0 & 22 & 15 \\
\hline U. de Concepción & 40 & 8 & 2 & 50 & 34 \\
\hline P.U. Católica & 10 & 1 & 0 & 11 & 7 \\
\hline U. de Santiago & 12 & 2 & 1 & 15 & 10 \\
\hline U. de Chile & 9 & 0 & 1 & 10 & 7 \\
\hline U. de los Lagos & 2 & 0 & 0 & 2 & 1 \\
\hline U. Austral & 2 & 1 & 1 & 4 & 3 \\
\hline U.C. Temuco (50\%) y U. la Frontera (50\%) & 2 & 0 & 0 & 2 & 1 \\
\hline U. de Chile $(70 \%)$ & 1 & 0 & 0 & 1 & 1 \\
\hline U. de Bío-Bío (70\%) y P.U. Católica (30\%) & 1 & 0 & 0 & 1 & 1 \\
\hline U. de Antofagasta & 11 & 1 & 0 & 12 & 8 \\
\hline U.T. Metropolitana & 1 & 0 & 0 & 1 & 1 \\
\hline U. de Magallanes & 1 & 0 & 0 & 1 & 1 \\
\hline U.T. F. Sta. María (50\%) y U. de Stgo. (50\%) & 3 & 0 & 0 & 3 & 2 \\
\hline U.C. de Valparaíso & 4 & 4 & 0 & 8 & 5 \\
\hline U. de Talca & 1 & 0 & 0 & 1 & 1 \\
\hline U. Mayor & 0 & 0 & 1 & 1 & 1 \\
\hline U. Santos Ossa & 0 & 1 & 0 & 1 & 1 \\
\hline U.C. del Norte & 0 & 0 & 1 & 1 & 1 \\
\hline U. del Bío-Bío & 1 & 0 & 0 & 1 & 1 \\
\hline P.U. Católica (50\%) y U. de Chile (50\%) & 1 & 0 & 0 & 1 & 1 \\
\hline TOTAL SOLICITUDES & 120 & 22 & 7 & 149 & 100 \\
\hline
\end{tabular}

Fuente: OFINTEC (Oficina de Información Tecnológica), Departamento de Propiedad Industrial del Ministerio de Economía.

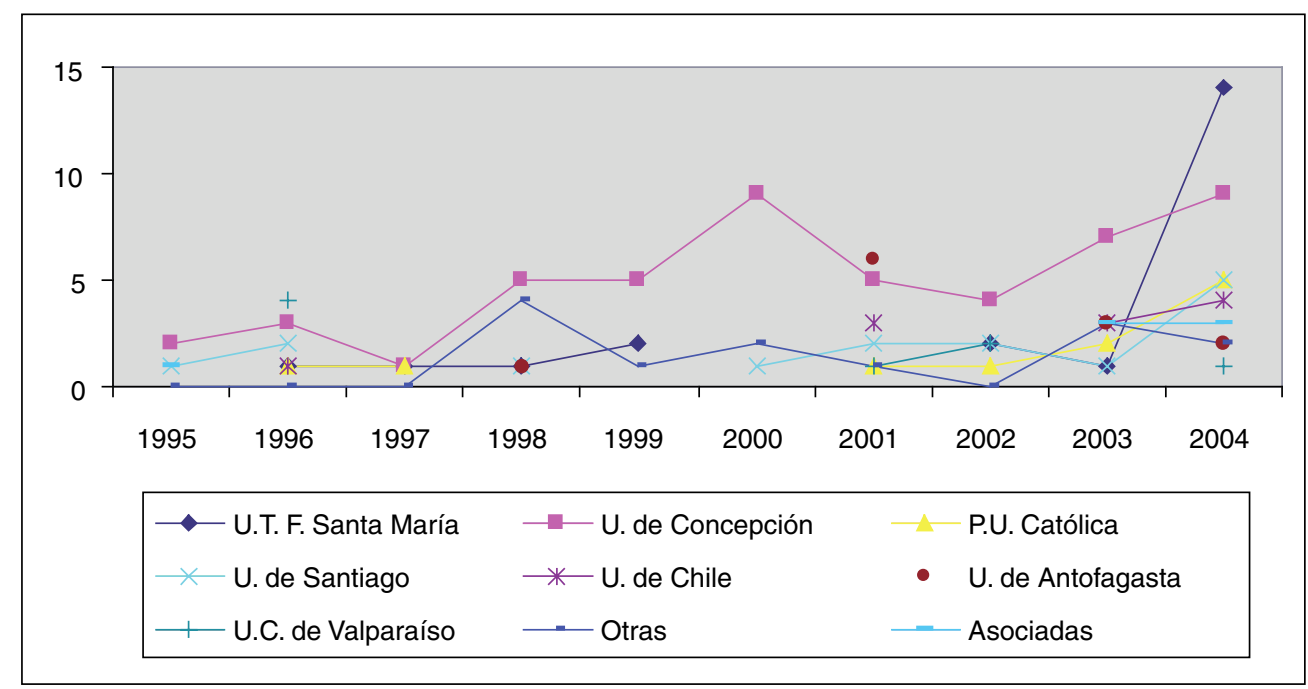

Fuente: Elaboración propia a partir de datos de OFINTEC.

Gráfico 4. Solicitudes de patentes universitarias en Chile tanto por las universidades individualmente como por varias universidades, las que se agruparon como asociadas. 
en Chile. La selección se realizó sobre la base de las solicitudes de patentes y a la información contenida en los sitios web que poseen las universidades, obteniéndose información de interés en lo referente a la gestión de la investigación, a su transferencia y difusión y a la gestión de los derechos de propiedad intelectual. No se incluyen la Universidad Católica del Norte y Universidad Mayor, a pesar de que tienen patentes concedidas porque en sus sitios web no hacen referencia alguna a sus políticas en materia de patentación.

Los sitios web visitados el 30 de agosto de 2005 fueron los siguientes:

- Universidad de Concepción www2.udec.cl/investigacion www2.udec.cl/upi

- Universidad Técnica Federico Santa María www.dgip.utfsm.cl

- Universidad de Chile www.investigacion.uchile.cl www.patentes.uchile.cl

- Universidad Austral www.uach.cl/direccion/investigacion

- Universidad Católica de Chile www.puc.cl/dipuc

- Universidad Tecnológica Metropolitana www.vtte.utem.cl

- Universidad Santiago de Chile www.vrid.usach.cl

- Universidad Católica de Valparaíso www.investigacion.ucv.cl www.ott.ucv.cl

\section{La Universidad de Concepción}

Con dos solicitudes de patentes concedidas en los últimos 10 años de 50 presentadas, posee un organismo, la Unidad de Propiedad Industrial (UPI), destinada a apoyar a todas las unidades que posee la universidad en materias relativas a la protección de la propiedad industrial e intelectual, así como en el comercio de los derechos que de ella deriven. Cuenta con un jefe de unidad, un asesor jurídico, dos profesionales y una secretaria. Las funciones que se le han definido son:

- Asesorar a reparticiones universitarias en materia de Propiedad Intelectual (PI) en sus diversos aspectos.

- Velar por los derechos de PI de la Universidad.

- Tramitar y prestar asesoría en solicitudes de PI.

- Capacitar a personal universitario en materias de PI.
- Coordinar la actividad de la universidad relacionada con la protección, explotación y comercio de PI.

- Promover asociaciones estratégicas.

- Crear estructuras jurídicas que permitan la obtención y explotación de los privilegios industriales.

- Prestar servicios a terceros en las áreas de su competencia.

Como consecuencia de las funciones definidas, presta servicios en los ámbitos de la tramitación de patentes de invención, modelos de utilidad y diseños industriales; la tramitación de marcas comerciales; los registros de propiedad intelectual, y la asesoría en asociaciones empresariales.

A la fecha ha suscrito diversos convenios de cooperación con distintas entidades que trabajan en el área de la protección de la propiedad industrial: con el Departamento de Propiedad Industrial del Ministerio de Economía; la Dirección de Bibliotecas, Archivos y Museos (DIBAM); el Servicio Agrícola y Ganadero (SAG) y la Cámara de la Producción y del Comercio de Concepción (CPCC).

Institucionalmente, la UPI participa, a través de su jefe de unidad, como miembro del Centro de Biotecnología de la Universidad; de la Subcomisión Marco Regulatorio de la Comisión Nacional de Biotecnología; de la Comisión Gobierno Regional de Integración Universidad-Empresa, y de la Oficina de Transferencia de Resultados de Investigación (O.T.R.I.).

Sus políticas de investigación incluyen estimular a los académicos para que los resultados de sus investigaciones sean publicados en revistas (ISI o de corriente principal), o que den origen a patentes. Como indicador de resultados incluye el número de patentes inscritas y aumentar la captación de financiamiento externo por la vía de licenciar tecnología. Como indicador de este objetivo se incluye el número de patentes concedidas.

\section{La Universidad de Chile}

Posee una Vicerrectoría de Investigación y Desarrollo cuyas políticas en esta materia apuntan a estrategias conducentes a la protección de derechos de patentes, al fomento de asociaciones transversales entre investigadores de diferentes unidades, a la asistencia a investigadores en el cumplimiento de normas legales y éticas y a la búsqueda proactiva de fuentes de financiamiento.

Para estos efectos, en el año 2003 creó una Comisión Central de Propiedad Industrial compuesta de cinco miembros titulares y tres suplentes designados por el Rector. Dentro de sus funciones se encuentran: 
- Proponer al Rector políticas universitarias en materia de propiedad industrial;

- Proponer los criterios y principios generales que permitan definir la procedencia y conveniencia de:

a) Solicitar una patente de invención o un modelo de utilidad;

b) Comercializar y/o ceder el uso de una patente de invención o un modelo de utilidad, a cualquier título.

Junto con la creación de la comisión se dictó un decreto (Decreto Universitario Exento $\mathrm{N}^{\circ} 0015.488$, de 20 de agosto de 2003) que incluye los procedimientos internos referidos a Patentes de Invención y Modelos de utilidad en los que se estipulan las acciones a seguir y las condiciones a cumplirse en estas materias. En el curso del presente año la comisión elaboró un Manual para la presentación de solicitudes de Patentes de Invención y Modelos de Utilidad que se encuentra disponible en el sitio web de la Universidad.

No obstante que según el Departamento de Propiedad Industrial en la última década a la universidad se le ha concedido una única patente en 1996, en su sitio web la Universidad de Chile da cuenta de 7 patentes de invención concedidas (www.patentes.uchile.cl). Esto puede explicarse si se trata de patentes concedidas en el extranjero.

\section{La Universidad Tecnológica Metropolitana}

Crea en 1994 la Vicerrectoría de Transferencia Tecnológica y Extensión (VTTE) con el propósito de promover la investigación y fomentar el conocimiento en la sociedad a través de instrumentos y políticas educativas y de desarrollo científico-tecnológico. Su misión es vincular a la universidad con su entorno mediante la prestación de servicios tecnológicos, la transferencia de innovaciones tecnológicas y la difusión de los conocimientos generales cultivados en las diferentes unidades académicas. Entre las unidades a su cargo se incluye una Oficina de Patentes y Marcas, destinada a proteger el conocimiento generado por los investigadores. Últimamente esta unidad, con el respaldo del programa bicentenario de ciencia y tecnología de CONICYT, ha abierto un concurso público de apoyo y fomento al patentamiento para la presentación de ideas innovadoras en el área de las ciencias de la ingeniería y diseño industrial. El objetivo que se persigue con este concurso es apoyar y gestionar mediante el financiamiento las ideas innovadoras presentadas que califiquen con los requerimientos establecidos por el Departamento de Propiedad Industrial, aporten al desarrollo industrial del país e integren personas/organizaciones nacionales a redes mundiales de producción tecnológica y científica.

\section{Universidad Católica de Chile}

En ésta el énfasis de sus políticas está puesto en la investigación en la frontera del conocimiento, la publicación de artículos en prestigiosas revistas internacionales, la implementación de programas académicos de nivel de excelencia para la formación de magíster y doctores en una variada gama de disciplinas, y docencia innovadora en pre y postgrado. Obsérvese que no existe ninguna mención explícita hacia las patentes. No obstante, en el seno de la Escuela de Ingeniería cuenta con una Dirección de Investigaciones Científicas y Tecnológicas (DICTUC), creada con miras a acercar el mundo académico a la realidad nacional por la vía de contribuir al proceso de transferencia tecnológica.

En 1994 DICTUC es transformada en una sociedad anónima con el ánimo de darle mayor flexibilidad desde el punto de vista empresarial y como una forma de desarrollar su misión de transferir la tecnología que se genera al interior de la Escuela de Ingeniería. En el 2004 se creó GeneraUC, destinada a apoyar la creación de empresas tecnológicas, la comercialización de tecnologías, el financiamiento de emprendimientos y la asesoría a empresas.

\section{Universidad Técnica Federico Santa María}

En el año 2004, fue la institución nacional que presentó mayor número de solicitudes de patentes de invención (14 solicitudes), seguida por la Corporación Nacional del Cobre (CODELCO) con 13 solicitudes, la Fábrica de envases Fosko S.A. (9 solicitudes) y la Universidad de Concepción (9 solicitudes). Esta universidad cuenta con una Dirección General de Investigación y Postgrado (DGIP), unidad que busca identificar aquellas iniciativas susceptibles de patentarse, para lo cual suele organizar jornadas destinadas a capacitar a los académicos respecto de los requisitos y etapas a seguir para solicitar patentes de invención.

\section{La Universidad Austral}

Posee una Dirección de Investigación y Desarrollo (DID) dependiente de la Vicerrectoría Académica, destinada a promover la investigación y el desarrollo por parte de los académicos, invitándolos a presentar iniciativas dentro de las modalidades de concurso para proyectos de investigación existentes. En materia de patentar - poner en valor- el nuevo conocimiento que se origina como resultado de investigaciones científico-tecnológicas, ha organizado un Seminario sobre Defensa de Propiedad Intelectual en el Marco de Proyectos FONDEF. Estos proyectos FONDEF incluyen, actualmente, una cláusula en 
la que se indica que todos los resultados de investigación y desarrollo en los que participa la universidad se deben proteger a nombre de ella. En febrero del presente año a la universidad le fue concedida la patente $\mathrm{N}^{\circ} 42351$ por un kit creado en el marco de un proyecto FONDEF.

\section{La Universidad de Santiago de Chile}

Desde el año 1988 cuenta con una Vicerrectoría de Investigación y Desarrollo (VRID), la que incluye como una de sus funciones la asociada a la gestión tecnológica. En el marco de la política en materia de investigación, entre sus objetivos no existe mención alguna al tema de las patentes, aunque 3 de sus 9 objetivos se pudieran relacionar con ellas, como son:

- Fomentar la vinculación universidad-empresa con la presentación de proyectos;

- Estimular la captación de recursos externos por la vía de las donaciones; y

- Estudiar la relación universidad-empresa a través de la gestión de proyectos de asignación directa.

Dependiente de la VRID se tiene al Departamento de Gestión Tecnológica (DGT), unidad orientada a la comunidad en general y al sector empresarial en particular, actuando en la identificación de oportunidades para la transferencia tecnológica que posibiliten el desarrollo de proyectos innovadores cuyos resultados generen productos tecnológicos susceptibles de ser comercializados por la propia universidad, empresas relacionadas u otras empresas creadas por la universidad.

Para generar vasos comunicantes entre dos mundos y culturas diferentes, en 1992 la universidad crea la Sociedad de Desarrollo Tecnológico (SDT), entidad privada, definida como una organización multidisciplinaria, orientada al mercado nacional e internacional, destinada a crear, desarrollar, coordinar, promover y apoyar actividades de transferencia tecnológica, asistencia técnica, educación continua y prestación de servicios técnicos desde la universidad hacia las empresas.

Además se cuenta con una fundación para el desarrollo empresarial (FUDE) cuya función es:

- Fomentar el desarrollo de proyectos conjuntos entre la universidad y las empresas;

- Difundir la existencia de instrumentos estatales y privados de apoyo al desarrollo tecnológico;

- Identificar y firmar convenios con entidades gremiales, empresariales u organizaciones vinculadas destinadas a trabajar en base a proyectos específicos;

- Apoyar la creación de empresas conjuntas entre la universidad y académicos con sectores privados;

- Apoyar la organización de actividades que permitan dar a conocer la oferta de la universidad y/o la demanda empresarial.

\section{Comparación con el desempeño de otros países}

Los antecedentes anteriores permiten realizar una breve comparación con lo observado en otros países. Especialmente importantes son las experiencias exitosas en períodos cualitativamente comparables. La primera comparación la hacemos respecto a EE.UU. en el período inmediatamente anterior y posterior a la promulgación de la Ley Bayh-Dole y la información se resume en el gráfico 5 .

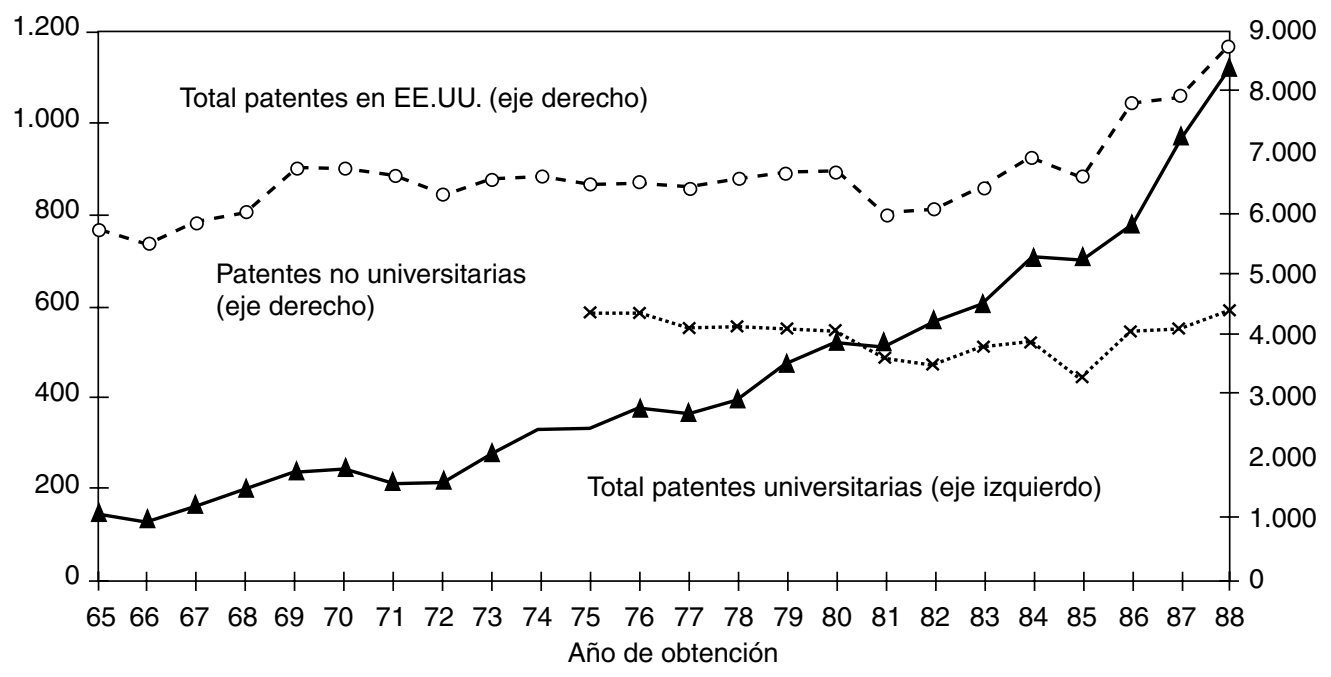

Gráfico 5. Patentes universitarias y totales en EE.UU. Fuente [16]. 
Observamos que el número de patentes totales en EE.UU. tenía una clara tendencia decreciente hasta principios de los 80. Aunque las patentes universitarias crecían sistemáticamente, dicho crecimiento no alcanzaba a compensar la declinación de la producción total de patentes. A partir de la promulgación de la ley Bayh-Dole en 1980 y sus modificaciones en 1982, las patentes universitarias aumentan notoriamente mientras el resto de la producción de patentes permanece estable. Sin embargo, el crecimiento de las patentes universitarias es tan elevado que influye positivamente en el total de patentes. Es posible apreciar que el crecimiento de la producción de patentes en EE.UU. desde mediados de los 80 se deriva exclusivamente del crecimiento de las patentes universitarias pues el resto de las patentes continúa una suave declinación. De hecho, en el período 1980/88 las patentes universitarias se multiplican por 2,5 veces.

En el caso europeo, la evolución de las patentes universitarias se aprecia en el gráfico 6.

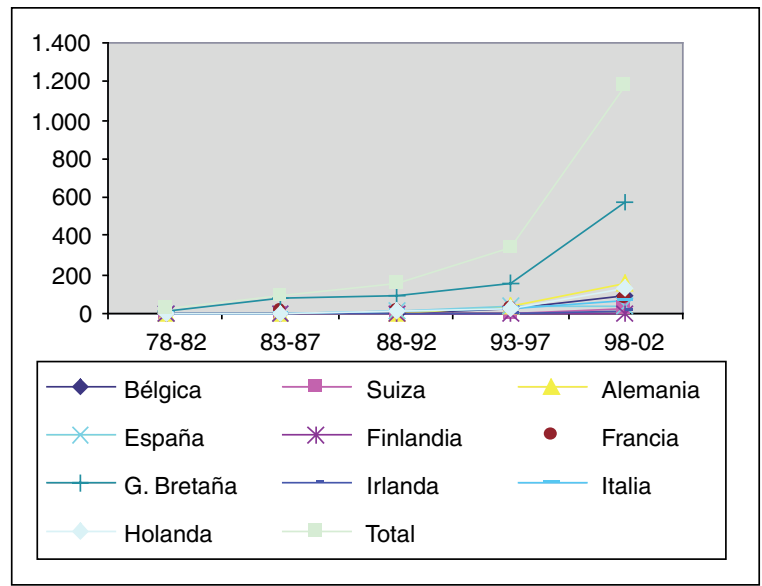

Fuente: Elaboración propia a partir de [9].

Gráfico 6. Patentes universitarias en países de la UE.

En Europa la incorporación de las universidades a la producción de tecnología patentada es algo posterior a lo acontecido en EE.UU. La producción de patentes universitarias europeas en el quinquenio 78/82 era excepcional y sólo unas pocas universidades británicas las producían y, por la misma razón, la proporción de patentes universitarias sobre el total de cada país en Europa resultaba insignificante.

Además en el caso europeo la incorporación de las universidades como productoras relevantes de patentes se hace por impulso de las legislaciones nacionales de cada país (posiblemente influenciadas por el éxito de Estados Unidos con la ley Bayh-Dole). Así la actividad patentadora se manifiesta inicialmente en Francia y Gran Bretaña a principios de los 80 , a fines de esa década en España e Italia, a mediados de los 90 en Bélgica, Alemania, Finlandia y Holanda y a fines de los 90 en Suiza e Irlanda. Además observamos que la proporción de la producción de patentes universitarias es notoriamente distinta en los países de la UE pues en países como España, Bélgica, Irlanda, Gran Bretaña y Holanda las universidades son productores relativos de patentes más importantes, pero no necesariamente los mayores productores absolutos de la UE.

Estas cifras muestran que a pesar de que las universidades europeas están sujetas a legislaciones nacionales, tradiciones académicas e institucionales y entornos económicos y empresariales heterogéneos, el resultado global es análogo al de EE.UU., una producción creciente en términos absolutos y relativos de la producción de patentes universitarias. Así pese a que la participación relativa de las universidades europeas es inferior a la de Estados Unidos a comienzos de los 80, la proporción llega a niveles parecidos a los de este país a fines de esa década.

\section{DISCUSIÓN}

Las estadísticas de solicitudes de patentes universitarias muestran que en los últimos 10 años ellas se han multiplicado por más de 10, desde 4 en 1995 hasta 45 en el año 2004. Sin embargo, pese a este incremento, sólo se han concedido siete patentes en dicho período, la última de ellas en 1999. Este resultado lleva a preguntar: ¿Por qué no se han concedido patentes en los últimos seis años? Se encuentran tres posibles respuestas:

1. Hay rezago en la oficina de patentes para procesar las solicitudes que llegan.

2. Las universidades tienen poca práctica patentadora, lo cual ocasiona una presentación inadecuada o inoportuna de la información de sus inventos ante la oficina de patentes.

3. El esfuerzo innovador de las universidades no se corresponde con los parámetros nacionales e internacionales.

El hecho que de las 149 solicitudes presentadas por las universidades en los últimos 10 años un $80 \%$ de ellas se encuentran en trámite, un $15 \%$ han sido abandonadas o desistidas y el resto corresponde a las concedidas, confirma la respuesta uno y en parte la dos. Esto implica que efectivamente existe un rezago en el trámite de las solicitudes por parte de la oficina de patentes, que, según la última fecha de aprobación, podría estimarse en seis años. 
Es importante tener en cuenta que estos datos también se explican como consecuencia de una realidad en las universidades marcada, entre otros factores, por centrar la evaluación de la $\mathrm{I}+\mathrm{D}$ en los resultados de ellas medidos por las publicaciones generadas antes que en las patentes generadas.

La obtención de patentes universitarias en Chile parece más bien anecdótica, lo cual permite interpretar que la contribución de las universidades a la producción de tecnología expresada en patentes industriales es indirecta, realizada a través de procesos de transferencia tecnológica (publicaciones, contratos tecnológicos, capacitación u otras modalidades) y también de la docencia.

En la actualidad los esfuerzos apuntan a dejar atrás una fase marcada por iniciativas individuales, aisladas de parte de los investigadores, sin mayor apoyo de instancias superiores, las que han tendido a privilegiar las publicaciones por sobre el patentamiento. Es así como actualmente, acicateadas por múltiples factores, las universidades, unas más que otras, se encuentran intentando pasar a una fase de institucionalización, de formalización, por la vía de la generación de unidades organizacionales específicas y de políticas explícitas en materia de propiedad intelectual.

Si se compara con las solicitudes de patentes de los países de la Unión Europea, los cuales aún no han asumido una política unificada hacia el impulso de los resultados de investigación producidos por las universidades, se encuentra que los resultados de la actividad patentadora en las universidades chilenas (lo cual se hace extensivo a América Latina) son realmente marginales. Ni qué decir si se compara con el número de solicitudes registradas y de solicitudes aprobadas por las universidades de Estados Unidos.

\section{CONCLUSIONES}

Las universidades que han destinado recursos de infraestructura, de recursos humanos y presupuestos para la gestión de derechos de propiedad intelectual y para la transferencia tecnológica, han obtenido resultados que van desde el incremento en la presentación de solicitudes como en la obtención de patentes, que aun siendo ellos escasos, no puede despreciarse la acumulación de experiencia y prestigio que dichas universidades obtienen con ello al posicionarse como pioneras en esta competitiva y exigente actividad. Además, debe reseñarse la importancia en la significativa participación del desarrollo científico y tecnológico del país a través de los proyectos FONDEF, en el período 1992-2002, destacándose la Universidad de Chile con 63 proyectos, la Universidad de Concepción con 53 y la Universidad Católica de Chile con 37 proyectos. La incapacidad para transformar estos proyectos, explícitamente orientados a la producción de tecnología, en patentes $u$ otras formas de propiedad industrial es un tema que debe investigarse con más detalle $[1,14]$.

Algunas universidades que han destinado recursos en infraestructura, recursos humanos y presupuestos en la transferencia tecnológica han priorizado frente a las patentes la difusión abierta del conocimiento, a través de publicaciones científicas, sin desmedro de su aporte científico técnico a la sociedad. Es el caso específico de la Universidad Católica de Chile.

Las ocho universidades analizadas han definido en su estructura una unidad para la gestión de la investigación de la transferencia tecnológica, y aunque generalmente no se ha asignado de manera explícita la gestión derechos de propiedad intelectual a estas dependencias, ellas lo tienen a su cargo [13].

Sin embargo, existe una heterogeneidad muy amplia en la definición de la misma unidad, en la dependencia a la cual pertenece, en la definición de sus objetivos y funciones, haciendo más difícil la comparación y caracterización de estas unidades, a diferencia de lo que ocurre en países como España donde se conformaron las OTRIS en todas las universidades, y aunque puedan tener algunas particularidades la homogeneización de su misión, funciones y políticas ha permitido el trabajo en red (existe la Red OTRIS) reduciendo costos en la creación de bases de datos, en los intercambios de información e incluso ha permitido la simplificación de estructuras con mayor incidencia en el sector productivo.

Respecto a la protección de la propiedad intelectual, cuatro universidades, a saber: la Universidad de Chile, Universidad de Concepción, Universidad Técnica Federico Santa María y Universidad Tecnológica Metropolitana expresan claramente su intencionalidad de tener, asistir o desarrollar las actividades referidas a la propiedad intelectual y entre ellas la patentación, esto es, como una manifiesta voluntad de entrar en la corriente jalonada por Estados Unidos a partir de la ley Bayh-Dole, de comercializar los resultados de la investigación, aun los financiados con dineros públicos para obtener una fuente de financiación alternativa. Sin embargo, consideramos que en los países subdesarrollados las universidades tienen mecanismos más efectivos para contribuir al desarrollo científico y tecnológico. Esto no quita que exploren la actividad patentadora, como ejercicio de aprendizaje y la consecución del prestigio. 
Los resultados observados en Chile deben calificarse de deficientes pues en el caso de EE.UU. después de la Bayh-Dole Act el número de patentes universitarias se multiplicó por más de 10 veces en los 10 años siguientes [10]; en el caso europeo los efectos son posteriores pero igualmente significativos. Las patentes de universidades europeas se multiplicaron "sólo" por 7,7 veces desde el quinquenio 1988/92 al 1998/02, aunque el punto de partida era muy inferior al caso norteamericano, pues en el quinquenio 1978/82 sólo había 20 patentes universitarias en Europa (17 de ellas en universidades de Gran Bretaña) [9] y en ambos casos la participación en relación a las producidas en cada conjunto de países de las patentes universitarias es creciente. En el caso de EE.UU., la proporción de patentes universitarias pasa de un $0,9 \%$ en 1980 a un $1,3 \%$ en 1988 y en el conjunto de la UE esta pasa de un $0,03 \%$ en el quinquenio $1988 / 92$ al $0,22 \%$ en el quinquenio $1998 / 02$.

Si bien se trata de aportes aparentemente menores, hay que recordar que las cifras no están controladas por importancia de las patentes, las cifras incluyen sólo a las patentes obtenidas por universidades en solitario (no incluyen asociaciones, participaciones minoritarias ni actividades contratadas a las universidades) y que se producen en contextos de producción importante en cada zona geográfica. De esa manera el desempeño de las universidades chilenas, 10 o 20 años después de las comparaciones indicadas, sólo puede calificarse de extremamente pobre.

Si además se consideran los esfuerzos estatales y la homogeneización del marco legal en el caso chileno, queda abierta la pregunta sobre las razones de este pobre resultado considerando que la actual legislación, de hecho, es de 1991, es decir, de hace casi 15 años.

El aporte de este trabajo se centra en cuantificar la realidad de la patentación en las universidades, observándose que el número de patentes universitarias concedidas es extremadamente bajo, no solo en términos absolutos, sino que en relación a las patentes nacionales concedidas. No obstante ello, se observa una tendencia al alza en los esfuerzos de patentación universitaria. Sin embargo, medidos en términos de patentes solicitadas no se corresponden con las patentes concedidas, por lo que, en términos de resultados, se puede afirmar que a la fecha los esfuerzos desplegados han sido infructuosos. Un trabajo a desarrollar debería centrarse en los factores de este diferencial entre el número de patentes solicitadas y concedidas, tanto desde las propias universidades como desde la unidad responsable de la concesión de patentes.
Adicionalmente este artículo abre la reflexión sobre la importancia de la patentación en las universidades como mecanismo de contribución directa al desarrollo tecnológico, pues el papel que deben jugar las universidades en el cambio tecnológico responde a unas exigencias históricas diferentes para contextos también diferentes en la evolución de la sociedad, pues, por una parte, cada vez es más borroso el límite entre la producción de ciencia y tecnología y, por otra, el rol que cumplen los actores sociales en dicha producción también es menos definido, estando el Estado, las universidades y las empresas en una interrelación permanente y en compromiso indistinto en la definición de políticas y estructuración de los sistemas nacionales o regionales de innovación, donde todos los actores participan en la formulación, financiación y ejecución de proyectos científico-tecnológicos.

Estimamos que parte del interés de este trabajo consiste en contribuir a formular hipótesis sobre las causas del bajo desempeño de las universidades chilenas, las que, no totalmente, deben encontrarse en la propia administración universitaria que aún no parece haber encontrado la manera de incorporar adecuadamente este aspecto del trabajo intelectual.

\section{AGRADECIMIENTOS}

Este trabajo ha sido desarrollado en el marco del proyecto 2003-4-157, "La Gestión del Conocimiento Comercial en las Universidades: Patentes y Licencias", con que el Programa de Cooperación Científica Internacional CONICYT/COLCIENCIAS ha apoyado al equipo de trabajo académico GESTOR (Gestión Tecnológica Organizacional).

\section{REFERENCIAS}

[1] P. Aghion and J. Tirole. "The Management of Innovation". Quarterly Journal of Economics. 109 (4), pp. 1185-1209. November 1994.

[2] P. Aghion and J. Tirole. "Opening the Black Box of Innovation”. European Economic Review. 38(3-4), pp. 701-10. April 1994.

[3] A. Agrawal and I. Cockburn. "The Anchor Tenant Hypothesis: Exploring the Role of Large, Local, R\&D-Intensive Firms in Regional Innovation Systems". International Journal of Industrial Organization. 21, pp. 1227-53. 2003. 
[4] D. Audretsch and P. Feldman. "R\&D Spillovers and the Geography of Innovation and Production". American Economic Review. 86 (3), pp. 630-40. June 1996.

[5] L. Branstetter. "Looking For International Knowledge Spillovers: A Review of the Literature with Suggestions for New Approaches". Annales d'Economie et de Statistique. N ${ }^{\circ} 49 / 50$, pp. 51740. 1998 .

[6] M. Callon. "Is Science a Public Good?" Science, Technology \& Human Values. 19, pp. 395-424. 1994.

[7] R.E. Caves, H. Crookel and J.P. Killing. "The Imperfect Market for Technology Licenses". Oxford Bulletin of Economics and Statistics. 45(3), pp. 249-67. August 1983.

[8] H.P. Ciapuscio. "El fuego de Prometeo: Tecnología y Sociedad”. Ed. EUDEBA. Buenos Aires, Argentina. 1994.

[9] G. Durán, A. Urraca, J.A. Negrín, N. Laguna, J. Díaz y A. Martín. “Análisis y Comparación de las Patentes Universitarias Españolas como Indicador de Resultados del Esfuerzo Investigador". Informe Proyecto $N^{\circ}$ Ea2003-0127. Ministerio de Educación y Cultura de España. Madrid, España. 2003.

[10] R. Henderson, J. Adam and M. Trajtenberg. "Universities as a Source of Commercial Technology: A Detailed Analysis of University Patenting 1965-1988”. NBER working Paper, No 5068. March 1995.

[11] A.B. Jaffe. "Real Effects of Academic Research". American Economic Review. 79 (5), pp. 957-70. December 1989.

[12] A.N. Link, J.T. Scott. U.S. "Science parks: The Diffusion of an Innovation and its Effects on the Academic Missions of Universities". International Journal of Industrial Organization. 21, pp. 132356. 2003.
[13] M. López, F. Cabrales, R. Schmal. "Gestión del Conocimiento: Una Revisión Teórica y su Asociación con la Universidad". Panorama Socioeconómico. Universidad de Talca. Vol. 23 $\mathrm{N}^{\mathrm{o}} 30$, pp. 20-30. 2005.

[14] K. Motohashi. "University-industry collaborations in Japan: The role of new technology-based firms in transforming the National Innovation System". Research Policy. Volume 34/5, pp. 583-96. June 2005.

[15] R. Nelson. "The Market Economy, and the Scientific Commons". Research Policy. 33, pp. 455-471. 2003.

[16] W.D. Nordhaus. "Invention, Growth and Welfare". Cambridge MA. MIT Press. 1969.

[17] "Patents and Innovation: Trends and Policy Challenges", OECD. Paris. 2004.

[18] L. Restrepo. "La Propiedad Intelectual de la Universidad ante el Tratado de Libre Comercio". Seminario Internacional de Gestión de la Innovación. Grupo GESTOR Universidad de Antioquia. Medellín. 2004.

[19] B.N. Sampt, D.C. Mowery and A.Z. Arvids. "Changes in University Patent Quality After the Bayh-Dole Act: A Re-Examination". International Journal of Industrial Organization. 21, pp. 137190. 2003.

[20] D.S. Siegel, P. Westhead and M. Wright. "Assessing the Impact of University Science Parks on research Productivity: Exploratory Firm-Level Evidence from the United Kingdom". International Journal of Industrial Organization. 21, pp. 1357-69. 2003.

[21] J.G. Thursby, G. Jerry and S. Kemp. "An Analysis of Productive Efficiency of University Commercialization Activities". Paper of Purdue University. 1999.

[22] L.G. Zucker, M.R. Darby and J. Armstrong. "Geographically Localized Knowledge: Spillovers or Markets?". Economic Inquiry, XXXVI, pp. 65-86. January 1998. 